\title{
Partial, Composite Fractional Operators, and Their Properties and Applications
}

\author{
Kaiying Deng ${ }^{1,2^{*}}$, Jingwei Deng ${ }^{2}$, and Suduo Li ${ }^{1}$ \\ ${ }^{1}$ College of Mathematics and Computer Science, Northwest Minzu University, Lanzhou 730124, China \\ ${ }^{2}$ Key Lab of Streaming Data Computing Technologies and Applications, Lanzhou 730030, China
}

\begin{abstract}
The paper discusses the properties of the partial fractional integrals, the partial fractional derivatives, and the composite fractional integrals and derivatives. Some basic formulas are derived; and the relations between the Riemann-Liouville and Caputo ones are also presented. Finally, as the specific application, a two dimensional Abel integral equation is solved.
\end{abstract}

\section{Introduction}

Fractional calculus, as a natural extension of the classical calculus, has the history of more than 300 years $[3,4,7,8,9]$. In fact, since the beginning of the theory of differential and integral calculus, the mathematicians began their investigations of the calculation of noninteger order derivatives and integrals. Most of the researchers of this topic cite 30th September 1695 as the birthday of the so called 'fractional calculus'. In a letter, dated 30th September 1695, L'Hopital wrote to Leibniz asking him a particular notation that he had used in his publication for the n-th derivative of a function $\frac{\mathrm{D}^{n} \mathrm{f}(\mathrm{k})}{\mathrm{Dx}^{n}}$; L'Hopital posed the question to Leibniz, what would the result be if $n=1 / 2$; Leibniz's response: “An apparent paradox, from which one day useful consequences will be drawn."

Following L'Hopital's and Liebniz's first inquisition, fractional calculus was primarily a study reserved for the best minds in mathematics. Fourier, Euler, Laplace are among the many that dabbled with fractional calculus and the mathematical consequences [7]. Many proposed, using their own notation and methodology, the definitions that fit the concept of noninteger order integral or derivative. The most famous of these definitions that have been popularized in the world of fractional calculus (not yet the world as a whole) are the Riemann-Liouville and Grunwald-Letnikov derivatives [6].

Most of the mathematical theories applicable to the study of fractional calculus were developed prior to the turn of the 20th century. However in the past 100 years, the widely engineering and scientific applications have been found. In some cases, the mathematics has to be made some changes to meet the requirements of physical reality. Caputo reformulated the more 'classic' definition of the Riemann-Liouville fractional derivative in order to conveniently specify the initial conditions. And the so called Caputo derivative was presented[2,3,4]. More recently, the space-time fractional operator was discussed [1].

In this paper, we introduce the partial fractional integral, partial fractional derivative, their compositions, and the relations between their Riemann-Liouville and Caputo versions. The properties of these operators are detailedly discussed; and a specific application of solving a two dimensional Abel integral equation is proposed. For easy of presentation, we restrict the left end point of the interval of the definition of fractional operators at ' 0 '; and the technique of Laplace transform is used [5].

\section{Partial Fractional Integrals And Their Compositions}

In our notation the Cauchy formula reads

$$
\mathrm{J}^{\mathrm{n}} \mathrm{f}(\mathrm{t}):=\mathrm{f}_{\mathrm{n}}(\mathrm{t})=\frac{1}{(\mathrm{n}-1) !} \int_{0}^{\mathrm{t}}(\mathrm{t}-\tau)^{\mathrm{n}-1} \mathrm{f}(\tau) \mathrm{d} \tau, \mathrm{t}>0, n \in \mathbb{N}
$$

where $\mathbb{N}$ is the set of positive integers. From this definition, it can be noted that $\mathrm{f}_{\mathrm{n}}(\mathrm{t})$ vanishes at $\mathrm{t}=0$ with its derivatives of order $1,2, \cdots, n-1$. Noting that $(n-$ $1) !=\Gamma(\mathrm{n})$, and introducing the arbitrary positive real number $\alpha$, one defines the fractional integral of order $\alpha>0$ :

$$
J^{\alpha} f(t):=\frac{1}{\Gamma(\alpha)} \int_{0}^{t}(t-\tau)^{\alpha-1} f(\tau) d \tau, t>0, \alpha \in R^{+} .
$$

Starting from the one dimensional case (2), we can naturally define the partial integral of the function $f(x, y)$ :

$$
\begin{aligned}
& J_{x}^{\alpha} f(x, y):=\frac{1}{\Gamma(\alpha)} \int_{0}^{x}(x-\tau)^{\alpha-1} f(\tau, y) d \tau, x>0, \alpha \in R^{+}, \\
& J_{y}^{\beta} f(x, y):=\frac{1}{\Gamma(\beta)} \int_{0}^{y}(y-\xi)^{\beta-1} f(x, \xi) d \xi, y>0, \beta \in R^{+},
\end{aligned}
$$

For the one-dimensional case (2), the semigroup property can be easily derived as 
So,

$$
\mathrm{J}^{\alpha} \mathrm{J}^{\beta}=\mathrm{J}^{\alpha+\beta}, \alpha, \beta \geq 0 .
$$

$$
\begin{aligned}
& \mathrm{J}_{\mathrm{x}}^{\alpha 1} \mathrm{~J}_{\mathrm{x}}^{\alpha 2}=\mathrm{J}_{\mathrm{x}}^{\alpha 1+\alpha 2}, \alpha_{1}+\alpha_{2} \geq 0 ; \\
& \mathrm{J}_{\mathrm{y}}^{\beta 1} \mathrm{~J}_{\mathrm{y}}^{\beta 2}=\mathrm{J}_{\mathrm{y}}^{\beta 1+\beta 2}, \beta_{1}+\beta_{2} \geq 0 ;
\end{aligned}
$$

It can be noted that the fractional integral can be considered as the Laplace convolution between $\Phi_{\alpha}(\mathrm{x})$ and $\mathrm{f}(\mathrm{x}, \mathrm{y})$, i.e.,

$$
\mathrm{J}_{\mathrm{x}}^{\alpha} \mathrm{f}(\mathrm{x}, \mathrm{y})=\Phi_{\alpha}(\mathrm{x}) * \mathrm{f}(\mathrm{x}, \mathrm{y}), \alpha>0 .
$$

Similarly, there exists

$$
\begin{gathered}
J_{\mathrm{y}}^{\beta} \mathrm{f}(\mathrm{x}, \mathrm{y})=\Phi_{\beta}(\mathrm{x}) * \mathrm{f}(\mathrm{x}, \mathrm{y}), \beta>0 ; \\
\mathrm{J}_{\mathrm{x}}^{\alpha} \mathrm{J}_{\mathrm{y}}^{\beta} \mathrm{f}(\mathrm{x}, \mathrm{y})=\Phi_{\alpha}(\mathrm{x}) *\left\{\Phi_{\beta}(\mathrm{y}) * \mathrm{f}(\mathrm{x}, \mathrm{y})\right\} \\
\quad=\Phi_{\alpha}(\mathrm{x}) * \Phi_{\beta}(\mathrm{y}) * \mathrm{f}(\mathrm{x}, \mathrm{y}), \alpha, \beta>0 ;
\end{gathered}
$$

and

$$
\begin{gathered}
\mathrm{J}_{\mathrm{x}}^{\alpha} \mathrm{J}_{\mathrm{y}}^{\beta} \mathrm{f}(\mathrm{x}, \mathrm{y})=\Phi_{\beta}(\mathrm{y}) *\left\{\Phi_{\alpha}(\mathrm{y}) * \mathrm{f}(\mathrm{x}, \mathrm{y})\right\} \\
=\Phi_{\beta}(\mathrm{y}) * \Phi_{\alpha}(\mathrm{x}) * \mathrm{f}(\mathrm{x}, \mathrm{y}), \alpha, \beta>0 .
\end{gathered}
$$

For the two-fold Laplace transforms, we have C; $\mathcal{L}_{\mathrm{y}} \mathcal{L}_{\mathrm{x}}\{\mathrm{f}(\mathrm{x}, \mathrm{y})\}=\mathcal{L}_{\mathrm{y}}\left\{\mathcal{L}_{\mathrm{x}} \mathrm{f}(\mathrm{x}, \mathrm{y})\right\}=\tilde{\mathrm{f}}^{\mathrm{yx}}\left(\mathrm{s}_{1}, \mathrm{~s}_{2}\right), \mathrm{s}_{1}, \mathrm{~s}_{2} \in$

C;

$\mathcal{L}_{\mathrm{x}} \mathcal{L}_{\mathrm{y}}\{\mathrm{f}(\mathrm{x}, \mathrm{y})\}=\mathcal{L}_{\mathrm{x}}\left\{\mathcal{L}_{\mathrm{y}} \mathrm{f}(\mathrm{x}, \mathrm{y})\right\}=\tilde{\mathrm{f}}^{\mathrm{xy}}\left(\mathrm{s}_{1}, \mathrm{~s}_{2}\right), \mathrm{s}_{1}, \mathrm{~s}_{2} \in$

Noting that

$$
\mathcal{L}_{\mathrm{x}}\left\{\Phi_{\alpha}(\mathrm{x})\right\}=\frac{1}{\mathrm{~s}_{1}^{\alpha}}, \mathrm{s}_{1} \in \mathrm{C} ;
$$

and

$$
\mathcal{L}_{\mathrm{y}}\left\{\Phi_{\beta}(\mathrm{y})\right\}=\frac{1}{\mathrm{~s}_{2}^{\beta}}, \mathrm{s}_{2} \in \mathrm{C},
$$

see e.g., [5], and recalling the convolution theorems for Laplace transforms, we get the following rules for the Laplace transforms of the fractional integrals,

$$
\begin{aligned}
& \mathcal{L}_{\mathrm{x}}\left\{\mathrm{J}_{\mathrm{x}}^{\alpha} \mathrm{f}(\mathrm{x}, \mathrm{y})\right\}=\mathcal{L}_{\mathrm{x}}\left\{\Phi_{\alpha}(\mathrm{x}) * \mathrm{f}(\mathrm{x}, \mathrm{y})\right\}=\frac{\widetilde{\mathrm{fx}}\left(\mathrm{s}_{1}, \mathrm{y}\right)}{\mathrm{s}_{1}^{\alpha}}, \alpha>0 ; \\
& \mathcal{L}_{\mathrm{y}}\left\{\mathrm{J}_{\mathrm{y}}^{\beta} \mathrm{f}(\mathrm{x}, \mathrm{y})\right\}=\mathcal{L}_{\mathrm{y}}\left\{\Phi_{\beta}(\mathrm{y}) * \mathrm{f}(\mathrm{x}, \mathrm{y})\right\}=\frac{\widetilde{\mathrm{f}}\left(\mathrm{x}, \mathrm{s}_{2}\right)}{\mathrm{s}_{2}^{\beta}}, \beta>0,
\end{aligned}
$$

which are the straightforward generalization of the case with a $n$-fold repeated integral $(\alpha=n)$.

\section{Partial Fractional Derivatives}

We note that

$$
J_{x}^{n} D_{x}^{n} f(x, y)=f(x, y)-\sum_{k=0}^{n-1} f^{(k)}\left(0^{+}, y\right) \frac{x^{k}}{k !}, x>
$$

0 ,

$$
\begin{aligned}
\mathrm{J}_{\mathrm{y}}^{n} \mathrm{D}_{\mathrm{y}}^{n} \mathrm{f}(\mathrm{x}, \mathrm{y})= & \mathrm{f}(\mathrm{x}, \mathrm{y})-\sum_{\mathrm{k}=0}^{n-1} \mathrm{f}^{(\mathrm{k})}\left(\mathrm{x}, 0^{+}\right) \frac{\mathrm{y}^{\mathrm{k}}}{\mathrm{k} !}, \mathrm{y} \\
& >0 .
\end{aligned}
$$

One defines the partial fractional derivative

$$
\begin{aligned}
& \mathrm{D}_{\mathrm{x}}^{\alpha} \mathrm{f}(\mathrm{x}, \mathrm{y}):= \\
& \left\{\begin{array}{c}
\frac{\partial^{\mathrm{m}}}{\partial \mathrm{x}^{\mathrm{m}}}\left[\frac{1}{\Gamma(\mathrm{m}-\alpha)} \int_{0}^{\mathrm{x}} \frac{\mathrm{f}(\tau, \mathrm{y})}{(\mathrm{x}-\tau)^{\alpha+1-\mathrm{m}}} \mathrm{d} \tau\right], \mathrm{m}-1<\alpha<m, \\
\frac{\partial^{\mathrm{m}}}{\partial \mathrm{x}^{\mathrm{m}}} \mathrm{f}(\mathrm{x}, \mathrm{y}), \alpha=\mathrm{m} ;
\end{array}\right.
\end{aligned}
$$

$$
\begin{aligned}
& \mathrm{D}_{\mathrm{y}}^{\beta} \mathrm{f}(\mathrm{x}, \mathrm{y}):= \\
& \left\{\begin{aligned}
\frac{\partial^{n}}{\partial \mathrm{y}^{n}}\left[\frac{1}{\Gamma(n-\beta)} \int_{0}^{\mathrm{y}} \frac{\mathrm{f}(\mathrm{x}, \xi)}{(\mathrm{y}-\xi)^{\beta+1-n}} \mathrm{~d} \xi\right], n-1<\beta<n, \\
\frac{\partial^{\mathrm{n}}}{\partial \mathrm{y}^{\mathrm{n}}} \mathrm{f}(\mathrm{x}, \mathrm{y}), \beta=n .
\end{aligned}\right.
\end{aligned}
$$

In fact, $\mathrm{D}_{\mathrm{x}}^{0}=\mathrm{J}_{\mathrm{x}}^{0}=\mathrm{I}$ and $\mathrm{D}_{\mathrm{y}}^{0}=\mathrm{J}_{\mathrm{y}}^{0}=\mathrm{I}$. Then we can easily recognize that

$$
\begin{aligned}
& D_{x}^{\alpha} J_{x}^{\alpha}=I, \alpha \geq 0 ; \\
& D_{y}^{\beta} J_{y}^{\beta}=I, \beta \geq 0 ; \\
& D_{x}^{\alpha} \neq I, \alpha>0 ; \\
& J_{y}^{\beta} D_{y}^{\beta} \neq I, \beta>0 ;
\end{aligned}
$$$$
\mathrm{J}_{\mathrm{X}}^{\alpha} \mathrm{D}_{\mathrm{X}}^{\alpha} \neq \mathrm{I}, \alpha>0 \text {; }
$$

And

$$
\begin{aligned}
& \mathrm{D}_{\mathrm{x}}^{\alpha} \mathrm{x}^{\gamma}=\frac{\Gamma(\gamma+1)}{\Gamma(\gamma+1-\alpha)} \mathrm{x}^{\gamma-\alpha}, \alpha>0, \gamma>-1, t>0 \\
& \mathrm{D}_{\mathrm{y}}^{\beta} \mathrm{y}^{\gamma}=\frac{\Gamma(\gamma+1)}{\Gamma(\gamma+1-\beta)} \mathrm{y}^{\gamma-\beta}, \beta>0, \gamma>-1, t>0
\end{aligned}
$$

We can get the Caputo partial fractional derivatives:

$$
:=\left\{\begin{array}{c}
\frac{1}{\Gamma(m-\alpha)} \int_{0}^{\mathrm{t}} \frac{\mathrm{f}_{\mathrm{x}}^{(m)}(\tau, \mathrm{y})}{(\mathrm{x}-\tau)^{\alpha+1-m}} \mathrm{~d} \tau, m-1<\alpha<m, \\
\frac{\partial^{m}}{\partial \mathrm{x}^{m}} \mathrm{f}(\mathrm{x}, \mathrm{y}), \alpha=m ;
\end{array}\right.
$$

and

$$
\begin{aligned}
& \mathrm{D}_{\mathrm{y}}^{\beta} \mathrm{f}(\mathrm{x}, \mathrm{y}):= \\
& \left\{\begin{array}{c}
\left.\frac{1}{\Gamma(n-\beta)} \int_{0}^{\mathrm{t}} \frac{\mathrm{f}_{\mathrm{x}}^{(n)}(\mathrm{x}, \xi)}{(\mathrm{y}-\xi)^{\beta+1-n}} \mathrm{~d} \xi\right], n-1<\beta<n, \\
\frac{\partial^{n}}{\partial \mathrm{y}^{n}} \mathrm{f}(\mathrm{x}, \mathrm{y}), \beta=n,
\end{array}\right. \\
& \text { where } \quad \mathrm{f}_{\mathrm{x}}^{(m)} \mathrm{f}(\mathrm{x}, \mathrm{y}):=\left.\frac{\partial^{m}}{\partial \mathrm{x}^{m}} \mathrm{f}(\mathrm{x}, \mathrm{y})\right|_{\mathrm{x}=\tau}
\end{aligned}
$$
$\mathrm{f}_{\mathrm{y}}^{(n)} \mathrm{f}(\mathrm{x}, \xi):=\left.\frac{\partial^{n}}{\partial \mathrm{y}^{n}} \mathrm{f}(\mathrm{x}, \mathrm{y})\right|_{\mathrm{y}=\xi}$, respectively. These definitions are of course restrictive; since they require the absolute integrability of the partial derivative of order $\mathrm{m}$ and $\mathrm{n}$, respectively. Whenever using the operators $\mathrm{D}_{\mathrm{x}}^{\alpha}$ and $\mathrm{D}_{\mathrm{y}}^{\beta}$, we implicitly assume that these conditions are met. It can be easily recognized that in general

$$
\begin{aligned}
& D_{x}^{\alpha} f(x, y):=D_{x}^{m} J_{x}^{m-\alpha} f(x, y) \neq J_{x}^{m-\alpha} D_{x}^{m} f(x, y):= \\
& D_{x}^{\alpha} f(x, y) \\
& D_{y}^{\beta} f(x, y):=D_{y}^{n} J_{y}^{n-\beta} f(x, y) \neq J_{y}^{n-\beta} D_{y}^{n} f(x, y):= \\
& D_{y}^{\beta} f(x, y) ;
\end{aligned}
$$

unless the function $\mathrm{f}(\mathrm{x}, \mathrm{y})$ along with its first $\mathrm{m}-1$ partial derivatives about $\mathrm{xvanishes}$ at $\mathrm{x}=0^{+}$and its first $\mathrm{n}-1$ partial derivatives about $\mathrm{y}$ vanishes at $\mathrm{y}=0^{+}$, respectively. Furthermore, for $\mathrm{m}-1<\alpha<\mathrm{m}$ and $x>0$,

$$
\begin{gathered}
\mathrm{D}_{\mathrm{x}}^{\alpha} \mathrm{f}(\mathrm{x}, \mathrm{y})= \\
\mathrm{D}_{\mathrm{x}}^{\alpha} \mathrm{f}(\mathrm{x}, \mathrm{y})+\sum_{\mathrm{k}=0}^{m-1} \frac{\mathrm{x}^{\mathrm{k}-\alpha}}{\Gamma(k-\alpha+1)} \mathrm{f}_{\mathrm{x}}^{(\mathrm{k})}\left(0^{+}, \mathrm{y}\right) .
\end{gathered}
$$

Similarly, we get 
$\mathrm{D}_{\mathrm{y}}^{\beta} \mathrm{f}(\mathrm{x}, \mathrm{y})=\mathrm{D}_{\mathrm{y}}^{\beta} \mathrm{f}(\mathrm{x}, \mathrm{y})+\sum_{\mathrm{k}=0}^{n-1} \frac{\mathrm{y}^{\mathrm{k}-\beta}}{\Gamma(k-\beta+1)} \mathrm{f}_{\mathrm{y}}^{(\mathrm{k})}\left(\mathrm{x}, 0^{+}\right) ;$

and therefore, recalling the partial fractional derivative of the power functions (26-27),

$$
\begin{aligned}
& \left.D_{x}^{\alpha} f(x, y)-\sum_{k=0}^{m-1} \frac{x^{k}}{k !} f_{x}^{(k)}\left(0^{+}, y\right)\right)=D_{x}^{\alpha} f(x, y) \\
& \left.D_{y}^{\beta} f(x, y)-\sum_{k=0}^{n-1} \frac{y^{k}}{k !} f_{y}^{(k)}\left(x, 0^{+}\right)\right)=D_{y}^{\beta} f(x, y)
\end{aligned}
$$

In particular, according to these definitions, it can be noted that the partial Caputo fractional derivative of a constant is zero, i.e.,

$$
\begin{aligned}
& D_{x}^{\alpha} g_{1}(y)=g_{1}(y) D_{x}^{\alpha} 1 \equiv 0, \alpha>0 ; \\
& D_{y}^{\beta} g_{2}(x)=g_{2}(x) D_{y}^{\beta} 1 \equiv 0, \beta>0 .
\end{aligned}
$$

We now explore the differences between the two kinds of the partial fractional derivatives, the RiemannLiouville fractional partial derivative, and (28)-(29), the Caputo fractional partial derivative. Based on (26)-(27), we can derive that

$$
\begin{aligned}
& D_{x}^{\alpha} x^{\alpha-1} g_{1}(y)=g_{1}(y) D_{x}^{\alpha} x^{\alpha-1} \equiv 0, \alpha>0, t>0 ; \\
& D_{y}^{\beta} g_{2}(x) y^{\beta-1}=g_{2}(x) D_{y}^{\beta} y^{\beta-1} \equiv 0, \beta>0, t>0 .
\end{aligned}
$$

with $\mathrm{x}>0, y>0$ and $m-1<\alpha \leq m, n-1<\beta \leq$ $n, m \in \mathbb{N}, n \in \mathbb{N}$,

$$
\begin{aligned}
D_{x}^{\alpha} f(x, y) & =D_{x}^{\alpha} g(x, y) \Leftrightarrow f(x, y) \\
& =g(x, y)+\sum_{i=1}^{m} c_{i}(y) x^{\alpha-i} ; \\
D_{y}^{\beta} f(x, y) & =D_{y}^{\beta} g(x, y) \Leftrightarrow f(x, y) \\
& =g(x, y)+\sum_{j=1}^{n} d_{j}(x) y^{\beta-j} ; \\
D_{x}^{\alpha} f(x, y) & =D_{x}^{\alpha} g(x, y) \Leftrightarrow f(x, y) \\
& =g(x, y)+\sum_{i=1}^{m} c_{i}(y) x^{m-\alpha} ; \\
D_{y}^{\beta} f(x, y) & =D_{y}^{\beta} g(x, y) \Leftrightarrow f(x, y) \\
& =g(x, y)+\sum_{j=1}^{n} d_{j}(x) y^{n-\beta} .
\end{aligned}
$$

Incidentally, we note that (38)-(39) provides an instructive example to show why $\mathrm{D}^{\alpha}$ is not the rightinverse to $\mathrm{J}^{\alpha}$,

$\mathrm{J}_{\mathrm{x}}^{\alpha} \mathrm{D}_{\mathrm{x}}^{\alpha} \mathrm{x}^{\alpha-1} \equiv 0$, but $\mathrm{D}_{\mathrm{x}}^{\alpha} \mathrm{J}_{\mathrm{x}}^{\alpha} \mathrm{x}^{\alpha-1}=\mathrm{x}^{\alpha-1}, \alpha>0, x>0$,

$\mathrm{J}_{\mathrm{y}}^{\beta} \mathrm{D}_{\mathrm{y}}^{\beta} \mathrm{y}^{\beta-1} \equiv 0$, but $\mathrm{D}_{\mathrm{y}}^{\beta} \mathrm{J}_{\mathrm{y}}^{\beta} \mathrm{y}^{\beta-1}=\mathrm{y}^{\beta-1}, \beta>0, y>0$. (45)

For the two definitions we also note a difference with respect to the formal limit as $\alpha \rightarrow(m-1)^{+}$. From (36)(37) and (33)-(34), there exist

$$
\lim _{\alpha \rightarrow(m-1)^{+}} D_{\mathrm{x}}^{\alpha} \mathrm{f}(\mathrm{x}, \mathrm{y})=\mathrm{D}_{\mathrm{x}}^{m} J_{\mathrm{x}}^{1} \mathrm{f}(\mathrm{x}, \mathrm{y})=\mathrm{D}_{\mathrm{x}}^{m-1} \mathrm{f}(\mathrm{x}, \mathrm{y}) ;
$$

$$
\begin{aligned}
\lim _{\beta \rightarrow(n-1)^{+}} D_{\mathrm{y}}^{\beta} \mathrm{f}(\mathrm{x}, \mathrm{y}) & =\mathrm{D}_{\mathrm{y}}^{n} \mathrm{~J}_{\mathrm{y}}^{1} \mathrm{f}(\mathrm{x}, \mathrm{y})=\mathrm{D}_{\mathrm{y}}^{n-1} \mathrm{f}(\mathrm{x}, \mathrm{y}) \\
\lim _{\alpha \rightarrow(m-1)^{+}} \mathrm{D}_{\mathrm{x}}^{\alpha} \mathrm{f}(\mathrm{x}, \mathrm{y}) & =\mathrm{J}_{\mathrm{x}}^{1} \mathrm{D}_{\mathrm{x}}^{m} \mathrm{f}(\mathrm{x}, \mathrm{y}) \\
& =\mathrm{D}_{\mathrm{x}}^{m-1} \mathrm{f}(\mathrm{x}, \mathrm{y})-\mathrm{f}_{\mathrm{x}}^{(m-1)} \\
\lim _{\beta \rightarrow(n-1)^{+}} \mathrm{D}_{\mathrm{y}}^{\beta} \mathrm{f}(\mathrm{x}, \mathrm{y}) & =\mathrm{J}_{\mathrm{y}}^{1} \mathrm{D}_{\mathrm{y}}^{n} \mathrm{f}(\mathrm{x}, \mathrm{y}) \\
& =\mathrm{D}_{\mathrm{y}}^{n-1} \mathrm{f}(\mathrm{x}, \mathrm{y})-\mathrm{f}_{\mathrm{y}}^{(n-1)}\left(\mathrm{x}, 0^{+}\right) .
\end{aligned}
$$

For the standard partial fractional derivative $\mathrm{D}_{\mathrm{x}}^{\alpha}$ or $D_{y}^{\beta}$, the Laplace transform, assumed to exist, requires the knowledge of the (bounded) initial values of the partial fractional integral $\mathrm{J}^{m-\alpha}$ and of its integer derivatives of order $\mathrm{k}=1,2, m-1$ or $n-1$ [8]. The corresponding rule reads

$$
\begin{gathered}
\mathcal{L}_{\mathrm{x}}\left\{\mathrm{D}_{\mathrm{x}}^{\alpha} \mathrm{f}(\mathrm{x}, \mathrm{y})\right\}=\mathrm{s}_{1}^{\alpha} \widetilde{\mathrm{x}}_{\mathrm{x}}\left(\mathrm{s}_{1}, \mathrm{y}\right) \\
\quad-\sum_{\mathrm{k}=0}^{m-1} \mathrm{D}_{\mathrm{x}}^{\mathrm{k}} \mathrm{J}_{\mathrm{x}}^{(m-\alpha)} \mathrm{f}\left(0^{+}, \mathrm{y}\right) \mathrm{s}_{1}^{m-1-\mathrm{k}}, \\
m-1<\alpha \leq m ; \\
\mathcal{L}_{\mathrm{y}}\left\{\mathrm{D}_{\mathrm{y}}^{\beta} \mathrm{f}(\mathrm{x}, \mathrm{y})\right\}= \\
\mathrm{s}_{2}^{\beta} \widetilde{\mathrm{f}}_{\mathrm{y}}\left(\mathrm{x}, \mathrm{s}_{2}\right)-\sum_{\mathrm{k}=0}^{n-1} \mathrm{D}_{\mathrm{y}}^{\mathrm{k}} \mathrm{J}_{\mathrm{y}}^{(n-\beta)} \mathrm{f}\left(\mathrm{x}, 0^{+}\right) \mathrm{s}_{2}^{n-1-\mathrm{k}}, \\
\quad n-1<\beta \leq n .
\end{gathered}
$$

In fact,

$$
\begin{aligned}
& \mathrm{J}_{\mathrm{x}}^{\alpha} \mathrm{D}_{\mathrm{x}}^{\alpha} \mathrm{f}(\mathrm{x}, \mathrm{y})=\mathrm{f}(\mathrm{x}, \mathrm{y})-\sum_{\mathrm{k}=0}^{m-1} \mathrm{f}_{\mathrm{x}}^{(\mathrm{k})}\left(0^{+}, \mathrm{y}\right) \frac{\mathrm{x}^{\mathrm{k}}}{\mathrm{k} !} ; \\
& \mathrm{J}_{\mathrm{y}}^{\beta} \mathrm{D}_{\mathrm{y}}^{\beta} \mathrm{f}(\mathrm{x}, \mathrm{y})=\mathrm{f}(\mathrm{x}, \mathrm{y})-\sum_{\mathrm{k}=0}^{n-1} \mathrm{f}_{\mathrm{y}}^{(\mathrm{k})}\left(\mathrm{x}, 0^{+}\right) \frac{\mathrm{y}^{\mathrm{k}}}{\mathrm{k} !} ;
\end{aligned}
$$

We can prove the following rule for the Laplace transform,

$$
\begin{aligned}
& \mathcal{L}_{\mathrm{x}}\left\{\mathrm{D}_{\mathrm{x}}^{\alpha} \mathrm{f}(\mathrm{x}, \mathrm{y})\right\} \\
&=\mathrm{s}_{1}^{\alpha} \tilde{\mathrm{f}}^{\mathrm{x}}(\mathrm{x}, \mathrm{y})-\sum_{\mathrm{k}=0}^{m-1} \mathrm{f}_{\mathrm{x}}^{(\mathrm{k})}\left(0^{+}, \mathrm{y}\right) \mathrm{s}_{1}^{\alpha-1-\mathrm{k}}, \\
& m-1<\alpha \leq m ; \\
& \mathcal{L}_{\mathrm{y}}\left\{\mathrm{D}_{\mathrm{y}}^{\beta} \mathrm{f}(\mathrm{x}, \mathrm{y})\right\} \\
&=\mathrm{s}_{2}^{\beta} \tilde{\mathrm{f}}^{\mathrm{y}}(\mathrm{x}, \mathrm{y})-\sum_{\mathrm{k}=0}^{n-1} \mathrm{f}_{\mathrm{y}}^{(\mathrm{k})}\left(\mathrm{x}, 0^{+}\right) \mathrm{s}_{2}^{\beta-1-\mathrm{k}}, \\
& n-1<\beta \leq n .
\end{aligned}
$$

\section{Composite Fractional Operators}

In general, both the operators of fractional differentiation, $D_{x}^{\alpha}, D_{y}^{\beta}$ and $D_{x}^{\alpha}, D_{y}^{\beta}$, do not satisfy either the semigroup property, or the (weaker) commutative property. To show how the Law of Exponents does not necessarily hold for the standard fractional derivative, we provide two simple example (with power functions) for which 


$$
\begin{aligned}
& D_{\mathrm{x}}^{\alpha 1} \mathrm{D}_{\mathrm{x}}^{\alpha 2}=\mathrm{D}_{\mathrm{x}}^{\alpha 2} \mathrm{D}_{\mathrm{x}}^{\alpha 1} \neq \mathrm{D}_{\mathrm{x}}^{\alpha 1+\alpha 2} \\
& \mathrm{D}_{\mathrm{x}}^{\alpha 1} \mathrm{D}_{\mathrm{x}}^{\alpha 2} \neq \mathrm{D}_{\mathrm{x}}^{\alpha 2} \mathrm{D}_{\mathrm{x}}^{\alpha 1}=\mathrm{D}_{\mathrm{x}}^{\alpha 1+\alpha 2} .
\end{aligned}
$$

For example, let us take $\mathrm{f}(\mathrm{x}, \mathrm{y})=\mathrm{x}^{-1 / 2}$ and $\alpha_{1}=$ $\alpha_{2}=1 / 2$. Then, using (31), we get $\mathrm{D}_{\mathrm{x}}^{1 / 2} \mathrm{f}(\mathrm{x}, \mathrm{y}) \equiv$ $0, \mathrm{D}_{\mathrm{x}}^{1 / 2} \mathrm{D}_{\mathrm{x}}^{1 / 2} \mathrm{f}(\mathrm{x}, \mathrm{y}) \equiv 0, \quad$ but $\quad \mathrm{D}_{\mathrm{x}}^{1 / 2+1 / 2} \mathrm{f}(\mathrm{x}+\mathrm{y})=$ $\operatorname{Df}(x, y)=-x^{-3 / 2} / 2$. Another example, let us take $\mathrm{g}(\mathrm{x}, \mathrm{y})=\mathrm{x}^{1 / 2}$ and $\alpha_{1}=1 / 2, \alpha_{2}=3 / 2$. Then, again using (42), we get $D_{x}^{1 / 2} g(x, y)=\sqrt{\pi} / 2, D_{x}^{3 / 2} g(x, y) \equiv 0$, but $\quad D_{x}^{1 / 2} D_{x}^{3 / 2} g(x, y) \equiv 0, D_{x}^{3 / 2} D_{x}^{1 / 2} g(x, y)=-t^{3 / 2} / 4$ and $D_{x}^{1 / 2+3 / 2} g(x, y)=D_{x}^{2} g(x, y)=-x^{3 / 2} / 4$. By the same way, we can obtain

$$
D_{y}^{\beta 1} D_{y}^{\beta 2} \neq D_{y}^{\beta 2} D_{y}^{\beta 1}, \beta_{1}, \beta_{2}>0 .
$$

it can be easily recognized that

$$
\begin{aligned}
\mathrm{D}_{\mathrm{x}}^{\mathrm{m} 1} \mathrm{D}_{\mathrm{x}}^{\mathrm{m} 2}= & \mathrm{D}_{\mathrm{x}}^{\mathrm{m} 2} \mathrm{D}_{\mathrm{x}}^{\mathrm{m} 1}, \mathrm{D}_{\mathrm{y}}^{\mathrm{n} 1} \mathrm{D}_{\mathrm{y}}^{\mathrm{n} 2} \\
= & \mathrm{D}_{\mathrm{y}}^{\mathrm{n} 2} \mathrm{D}_{\mathrm{y}}^{\mathrm{n} 1}, \mathrm{n}_{1}, \mathrm{n}_{2}, \mathrm{~m}_{1}, \mathrm{~m}_{2} \in \mathbb{N} ; \\
& \mathrm{J}_{\mathrm{x}}^{\alpha} \mathrm{J}_{\mathrm{y}}^{\beta}=\mathrm{J}_{\mathrm{y}}^{\beta} J_{\mathrm{x}}^{\alpha}, \alpha, \beta \geq 0 ; \\
& \mathrm{D}_{\mathrm{y}}^{\mathrm{n}} \mathrm{J}_{\mathrm{x}}^{\alpha}=\mathrm{J}_{\mathrm{x}}^{\alpha} \mathrm{D}_{\mathrm{y}}^{\mathrm{n}}, \alpha \geq 0, \mathrm{n} \in \mathbb{N} ; \\
& \mathrm{D}_{\mathrm{x}}^{\mathrm{m}} \mathrm{J}_{\mathrm{y}}^{\beta}=\mathrm{J}_{\mathrm{y}}^{\beta} \mathrm{D}_{\mathrm{x}}^{\mathrm{m}}, \beta \geq 0, \mathrm{~m} \in \mathbb{N} .
\end{aligned}
$$

Therefore, we have

$$
\begin{aligned}
& D_{y}^{\beta} J_{x}^{\alpha}=D_{y}^{n} J_{y}^{n-\beta} J_{x}^{\alpha}=J_{x}^{\alpha} D_{y}^{\beta}, \alpha, \beta \geq 0 ; \\
& D_{x}^{\alpha} J_{y}^{\beta}=D_{x}^{m} J_{x}^{m-\alpha} J_{y}^{\beta}=J_{y}^{\beta} D_{x}^{\alpha}, \alpha, \beta \geq 0 .
\end{aligned}
$$

Hence, there are the following commutative properties for the fractional partial derivatives

$$
\begin{aligned}
& D_{y}^{\beta} D_{x}^{\alpha}=D_{y}^{n} J_{y}^{n-\beta} D_{x}^{m} J_{x}^{m-\alpha}=D_{x}^{\alpha} D_{y}^{\beta}, \alpha, \beta \geq 0 \\
& D_{y}^{\beta} D_{x}^{\alpha}=J_{y}^{n-\beta} D_{y}^{n} J_{x}^{m-\alpha} D_{x}^{m}=D_{x}^{\alpha} D_{y}^{\beta}, \alpha, \beta \geq 0 ;
\end{aligned}
$$

Considering that the Laplace transform is an integral transformation and noting (4)-(5), the following expression is valid,

$$
\mathcal{L}_{\mathrm{y}} \mathcal{L}_{\mathrm{x}}=\mathcal{L}_{\mathrm{x}} \mathcal{L}_{\mathrm{y}}
$$

i.e., $\tilde{\mathrm{f}}^{\mathrm{yx}}\left(\mathrm{s}_{1}, \mathrm{~s}_{2}\right)=\tilde{\mathrm{f}}^{\mathrm{xy}}\left(\mathrm{s}_{1}, \mathrm{~s}_{2}\right)$. What is more, according to (8)-(9) and the previous expressions, we can get

$$
\begin{gathered}
\mathcal{L}_{\mathrm{y}} \mathcal{L}_{\mathrm{x}} \mathrm{J}_{\mathrm{x}}^{\alpha} \mathrm{J}_{\mathrm{y}}^{\beta}\{\mathrm{f}(\mathrm{x}, \mathrm{y})\}=\mathcal{L}_{\mathrm{y}}\left\{\frac{1}{\mathrm{~s}_{1}^{\alpha}} \mathcal{L}_{\mathrm{x}}\left\{\mathrm{J}_{\mathrm{y}}^{\beta} \mathrm{f}(\mathrm{x}, \mathrm{y})\right\}\right\} \\
=\frac{1}{\mathrm{~s}_{1}^{\alpha} \mathrm{s}_{2}^{\beta}} \tilde{\mathrm{f}}^{\mathrm{yx}}\left(\mathrm{s}_{1}, \mathrm{~s}_{2}\right), \mathrm{s}_{1}, \mathrm{~s}_{2} \in \mathrm{C} ; \\
\mathcal{L}_{\mathrm{x}} \mathcal{L}_{\mathrm{y}} \mathrm{J}_{\mathrm{y}}^{\beta} \mathrm{J}_{\mathrm{x}}^{\alpha}\{\mathrm{f}(\mathrm{x}, \mathrm{y})\}=\mathcal{L}_{\mathrm{y}} \mathcal{L}_{\mathrm{x}} \mathrm{J}_{\mathrm{x}}^{\alpha} \mathrm{J}_{\mathrm{y}}^{\beta}\{\mathrm{f}(\mathrm{x}, \mathrm{y})\} \\
=\frac{1}{\mathrm{~s}_{1}^{\alpha} \mathrm{s}_{2}^{\beta}} \tilde{\mathrm{f}}^{\mathrm{xy}}\left(\mathrm{s}_{1}, \mathrm{~s}_{2}\right), \mathrm{s}_{1}, \mathrm{~s}_{2} \in \mathrm{C} .
\end{gathered}
$$

\section{Solving the Abel Integral Equation}

Here we limit ourselves to put some emphasis on the method of the Laplace transforms; and use the results given in the above sections to solve the equation. Let us consider the two dimensional Abel integral equation

$\frac{1}{\Gamma(\alpha)} \frac{1}{\Gamma(\beta)} \int_{0}^{\mathrm{x}} \frac{1}{(\mathrm{x}-\tau)^{1-\alpha}} \mathrm{d} \tau \int_{0}^{\mathrm{y}} \frac{\mathrm{u}(\tau, \xi)}{(\mathrm{y}-\xi)^{1-\beta}} \mathrm{d} \xi=\mathrm{f}(\mathrm{x}, \mathrm{y}), \alpha>0, \beta<$

1 ,

where $f(x, y)$ is a given function. By the notations of fractional integrals, there exists

$$
\mathrm{J}_{\mathrm{x}}^{\alpha} \mathrm{J}_{\mathrm{y}}^{\beta} \mathrm{u}(\mathrm{x}, \mathrm{y})=\mathrm{f}(\mathrm{x}, \mathrm{y}), \alpha>0, \beta<1 ;
$$

and consequently from the properties of partial fractional calculus we can get

$$
u(x, y)=D_{y}^{\beta} D_{x}^{\alpha} f(x, y)
$$

We can also solve (70) by using the Laplace transforms.

Noting from (68), there exists

$$
\mathcal{L}_{\mathrm{y}} \mathcal{L}_{\mathrm{x}}\left\{\mathrm{J}_{\mathrm{x}}^{\alpha} \mathrm{J}_{\mathrm{y}}^{\beta} \mathrm{u}(\mathrm{x}, \mathrm{y})\right\}=\mathcal{L}_{\mathrm{y}} \mathcal{L}_{\mathrm{x}}\{\mathrm{f}(\mathrm{x}, \mathrm{y})\},
$$

i.e.,

$$
\frac{1}{\mathrm{~s}_{1}^{\alpha} \mathrm{s}_{2}^{\beta}} \tilde{\mathrm{u}}^{\mathrm{yx}}\left(\mathrm{s}_{1}, \mathrm{~s}_{2}\right)=\tilde{\mathrm{f}}^{\mathrm{yx}}\left(\mathrm{s}_{1}, \mathrm{~s}_{2}\right) \text {. }
$$

Now we can choose two different ways to get the inverse Laplace transform from (74). Writing (74) as

$$
\begin{gathered}
\frac{1}{s_{1}} \tilde{u}^{\mathrm{yx}}\left(\mathrm{s}_{1}, \mathrm{~s}_{2}\right)=\mathrm{s}_{1}^{\alpha-1} \mathrm{~s}_{2}^{\beta} \tilde{\mathrm{f}}^{\mathrm{yx}}\left(\mathrm{s}_{1}, \mathrm{~s}_{2}\right) ; \\
\mathrm{J}_{\mathrm{x}}^{1} \tilde{\mathrm{u}}^{\mathrm{y}}\left(\mathrm{x}, \mathrm{s}_{2}\right)=\mathrm{J}_{\mathrm{x}}^{1-\alpha} \mathrm{s}_{2}^{\beta} \tilde{\mathrm{f}}^{\mathrm{y}}\left(\mathrm{x}, \mathrm{s}_{2}\right) ; \\
\tilde{\mathrm{u}}^{\mathrm{y}}\left(\mathrm{x}, \mathrm{s}_{2}\right)=\mathrm{D}_{\mathrm{x}}^{1} \mathrm{~J}_{\mathrm{x}}^{1-\alpha} \mathrm{J}_{\mathrm{x}}^{1-\alpha} \mathrm{s}_{2}^{\beta} \tilde{\mathrm{f}}^{\mathrm{y}}\left(\mathrm{x}, \mathrm{s}_{2}\right)=\mathrm{s}_{2}^{\beta} \mathrm{D}_{\mathrm{x}}^{\alpha} \tilde{\mathrm{f}}^{\mathrm{y}}\left(\mathrm{x}, \mathrm{s}_{2}\right) ; \\
\mathrm{J}_{\mathrm{y}}^{1} \mathrm{u}(\mathrm{x}, \mathrm{y})=\mathrm{J}_{\mathrm{y}}^{1-\beta} \mathrm{D}_{\mathrm{x}}^{\alpha} \mathrm{f}(\mathrm{x}, \mathrm{y}) ;
\end{gathered}
$$

we obtain

$$
u(x, y)=D_{y}^{\beta} D_{x}^{\alpha} f(x, y)=D_{y}^{1} J_{y}^{1-\beta} D_{x}^{\alpha} f(x, y) .
$$

On the other hand, writing (95) as

$$
\begin{array}{r}
\tilde{\mathrm{u}}^{\mathrm{yx}}\left(\mathrm{s}_{1}, \mathrm{~s}_{2}\right)=\frac{1}{\mathrm{~s}_{1}^{1-\alpha}}\left[\begin{array}{c}
\left.\left.\mathrm{s}_{1}\left\{\mathrm{~s}_{2}^{\beta} \tilde{\mathrm{f}}^{\mathrm{yx}}\left(\mathrm{s}_{1}, \mathrm{~s}_{2}\right)\right\}-\right]+\frac{\mathrm{s}_{2}^{\beta} \tilde{\mathrm{f}}^{\mathrm{y}}\left(0^{+}, \mathrm{s}_{2}\right)}{\mathrm{s}_{1}^{1-\alpha}} ; \mathrm{s}_{2}^{\beta} \tilde{\mathrm{f}}^{\mathrm{y}}\left(0^{+}, \mathrm{s}_{2}\right)\right\}
\end{array}\right] \\
\mathcal{L}_{\mathrm{x}} \tilde{\mathrm{u}}^{\mathrm{y}}\left(\mathrm{x}, \mathrm{s}_{2}\right)= \\
\mathcal{L}_{\mathrm{x}}\left\{\Phi_{1-\alpha}(\mathrm{x}) * \mathrm{D}_{\mathrm{x}}^{1} \mathrm{~s}_{2}^{\beta} \tilde{\mathrm{f}}^{\mathrm{y}}\left(\mathrm{x}, \mathrm{s}_{2}\right)\right\} \\
+\mathcal{L}_{\mathrm{x}}\left\{\tilde{\mathrm{f}}^{\mathrm{y}}\left(0^{+}, \mathrm{s}_{2}\right) \Phi_{1-\alpha}(\mathrm{x})\right\} ;
\end{array}
$$

leads to

$$
\mathcal{L}_{\mathrm{y}} \mathrm{u}(\mathrm{x}, \mathrm{y})=\mathrm{D}_{\mathrm{x}}^{\alpha}\left\{\mathrm{s}_{2}^{\beta} \tilde{\mathrm{f}}^{\mathrm{y}}\left(\mathrm{x}, \mathrm{s}_{2}\right)\right\}+\tilde{\mathrm{f}}^{\mathrm{y}}\left(0^{+}, \mathrm{s}_{2}\right) \Phi_{1-\alpha}(\mathrm{x}) .
$$

Hence, we can ge

$$
\begin{gathered}
u(x, y)=D_{y}^{\beta} D_{x}^{\alpha} f(x, y)+\Phi_{1-\beta}(y) D_{x}^{\alpha} f\left(x, 0^{+}\right) \\
+\Phi_{1-\alpha}(x) D_{y}^{\beta} f\left(0^{+}, y\right) \Phi_{1-\alpha}(x) \Phi_{1-\beta}(y) f\left(0^{+}, 0^{+}\right) ;
\end{gathered}
$$

$\mathrm{D}_{\mathrm{x}}^{\alpha} \mathrm{f}(\mathrm{x}, \mathrm{y})=\mathrm{D}_{\mathrm{x}}^{\alpha} \mathrm{f}(\mathrm{x}, \mathrm{y})+\sum_{\mathrm{k}=0}^{m-1} \frac{\mathrm{x}^{\mathrm{k}-\alpha}}{\Gamma(\mathrm{k}-\alpha+1)} \mathrm{f}_{\mathrm{x}}^{(\mathrm{k})}\left(0^{+}, \mathrm{y}\right)$;

$$
\mathrm{D}_{\mathrm{y}}^{\beta} \mathrm{f}(\mathrm{x}, \mathrm{y})=\mathrm{D}_{\mathrm{y}}^{\beta} \mathrm{f}(\mathrm{x}, \mathrm{y})+\sum_{\mathrm{k}=0}^{n-1} \frac{\mathrm{y}^{\mathrm{k}-\beta}}{\Gamma(\mathrm{k}-\beta+1)} \mathrm{f}_{\mathrm{y}}^{(\mathrm{k})}\left(\mathrm{x}, 0^{+}\right) \text {. }
$$

As a specific example, for $0<\alpha<1$, there exists

$$
\begin{aligned}
& D_{x}^{\alpha} f(x, y)=D_{x}^{\alpha} f(x, y)+\Phi_{1-\alpha}(x) f\left(0^{+}, y\right) \\
& D_{y}^{\beta} f(x, y)=D_{y}^{\beta} f(x, y)+\Phi_{1-\beta}(y) f\left(x, 0^{+}\right)
\end{aligned}
$$

where we have used (52) with $m=1$ and (53) with $\mathrm{n}=1$.

$$
\begin{aligned}
& D_{y}^{\beta} D_{x}^{\alpha} f(x, y)=D_{y}^{\beta} D_{x}^{\alpha} f(x, y)+\Phi_{1-\beta}(y) D_{x}^{\alpha} f\left(x, 0^{+}\right) \\
& +\Phi_{1-\alpha}(x) D_{y}^{\beta} f\left(0^{+}, y\right)+\Phi_{1-\alpha}(x) \Phi_{1-\beta}(y) f\left(0^{+}, 0^{+}\right) .
\end{aligned}
$$

Thus, the solutions (78) and (82) are expressed in terms of the partial fractional derivatives $D_{y}^{\beta} D_{x}^{\alpha}$ and $D_{y}^{\beta} D_{x}^{\alpha}$. In fact, many of the above conclusions can be directly generalized to those of $n$-dimensional case, where $n \geq 2$. 


\section{Conclusions}

Since the beginning of the theory of differential and integral calculus, the mathematicians began their investigations of the calculation of non-integer order derivatives and integrals. In this paper, we introduce the properties of the partial fractional integrals, the partial fractional derivatives, and the composite fractional integrals and derivatives, and a specific application of solving a two dimensional Abel integral equation is proposed.

\section{Acknowledgments}

This work was supported by the Fundamental Research Funds for the Central Universities (Northwest Minzu University) under Grant No. 31920150039, the Northwest Minzu University Talent Research Funds (No.xbmuyjrc201632) and the Youth Science and Technology Foundation of Gansu Province (No. 1610RJYA109) and Fundamental Research Funds for the Central Universities (NO.31920170001, 31920170009, 31920170143).

\section{References}

1. B. Baeumer, M. Meerschaert, J. Mortensen, Proceedings of the American Mathematical Society, pp. 2273-2282, (2005)

2. M. Caputo, Elasticit à e Dissipazione, Zanichelli, Bologna, (1969)

3. K. Diethelm, Springer-Verlag, New York, (2010)

4. K. Diethelm, N. J. Ford, J. Math. Anal. Appl. 265 pp. 229-248,( 2002)

5. G. Doetsch, Berlin, Germany, (1974)

6. R. Gorenflo, F. Mainardi, Mathematics, 49(2), pp. 277-290, (2008)

7. K. Nishimoto, An essence of Nishimoto's Fractional Calculus, (Calculus in the 21st Century), Descartes Press Company, (1991)

8. K. B. Oldham, J. Spanier, New York, USA, (1974)

9. G. Samko, A. Kilbas, I. Marchev, Gordon and Breach Science Publishers, Singapore, (1993) 